\title{
The Join of the Varieties of R-trivial and L-trivial Monoids via Combinatorics on Words
}

\author{
Manfred Kufleitner $\| \dagger$ and Alexander Lausen!: \\ University of Stuttgart, Germany \\ received 10 ${ }^{\text {th }}$ January 2012, revised 25th May 2012, accepted 26 $6^{\text {th }}$ May 2012.
}

The join of two varieties is the smallest variety containing both. In finite semigroup theory, the varieties of $\mathscr{R}$-trivial and $\mathscr{L}$-trivial monoids are two of the most prominent classes of finite monoids. Their join is known to be decidable due to a result of Almeida and Azevedo. In this paper, we give a new proof for Almeida and Azevedo's effective characterization of the join of $\mathscr{R}$-trivial and $\mathscr{L}$-trivial monoids. This characterization is a single identity of $\omega$-terms using three variables.

Keywords: finite semigroup theory, join of pseudovarieties, Green's relations, combinatorics on words

\section{Introduction}

Green's relations $\mathscr{R}$ and $\mathscr{L}$ are a standard tool in the study of semigroups [5]. In the context of finite monoids, among other results, they have been used to give effective characterizations of language classes such as star-free languages [3, 11] and piecewise testable languages [6, 12]. A deterministic extension of piecewise testable languages yields the class of languages corresponding to $\mathscr{R}$-trivial monoids, and a codeterministic extension corresponds to $\mathscr{L}$-trivial monoids [4, 9].

Almeida and Azevedo gave an effective characterization for the least variety of finite monoids containing all $\mathscr{R}$-trivial and all $\mathscr{L}$-trivial monoids [2], i.e., for the join of the two varieties. Their proof is based on sophisticated algebraic techniques, on Reiterman's Theorem [10], and on a combinatorial result of König [7]. In this paper, we give a new proof of Almeida and Azevedo's Theorem. The current proof was inspired by another proof of the authors [8], which in turn uses ideas of Klíma [6]. The main ingredient is a system of congruences which relies on simple combinatorics on words.

\footnotetext{
${ }^{\dagger}$ Email: kufleitner@fmi.uni-stuttgart.de.

\#Email: lauser@fmi. uni-stuttgart.de 


\section{Preliminaries}

Let $A$ be a finite alphabet. The set of finite words over $A$ is denoted by $A^{*}$. It is the free monoid over $A$. The empty word is 1 . The content of a word $u=a_{1} \cdots a_{n}$ with $a_{i} \in A$ is $\alpha(u)=\left\{a_{1}, \ldots, a_{n}\right\}$, and its length is $|u|=n$. The length of the empty word is 0 . A word $u$ is a prefix (respectively suffix) of $v$ if there exists $x \in A^{*}$ such that $u x=v$ (respectively $x u=v$ ); if $x \neq 1$, then $u$ is a proper prefix.

For more details concerning the algebraic concepts introduced in the remainder of this section, we refer the reader to textbooks such as [1, 4, 9]. Green's relations $\mathscr{R}$ and $\mathscr{L}$ are important tools in the study of finite monoids. Let $M$ be a finite monoid. We set $u \mathscr{R} v$ for $u, v \in M$ if $u M=v M$, and the latter condition is equivalent to the existence of $x, y \in M$ with $u=v x$ and $v=u y$. Symmetrically, $u \mathscr{L} v$ if $M u=M v$. The monoid $M$ is $\mathscr{R}$-trivial (respectively $\mathscr{L}$-trivial) if $\mathscr{R}$ (respectively $\mathscr{L}$ ) is the identity relation on $M$. We write $u<\mathscr{R} v$ if $u M \subsetneq v M$, and we write $u<\mathscr{L} v$ if $M u \subsetneq M v$.

A variety of finite monoids is a class of monoids closed under finite direct products, submonoids, and quotients. A variety of finite monoids is often called a pseudovariety in order to distinguish from varieties in Birkhoff's sense. Since we do not need this distinction in the current paper, whenever we use the term variety we mean a variety of finite monoids. The join $\mathbf{V}_{1} \vee \mathbf{V}_{2}$ of two varieties $\mathbf{V}_{1}$ and $\mathbf{V}_{2}$ is the smallest variety containing $\mathbf{V}_{1} \cup \mathbf{V}_{2}$. A monoid $M$ is in $\mathbf{V}_{1} \vee \mathbf{V}_{2}$ if and only if there exist $M_{1} \in \mathbf{V}_{1}$ and $M_{2} \in \mathbf{V}_{2}$ such that $M$ is a quotient of a submonoid of $M_{1} \times M_{2}$. If $M$ is a finite monoid, then there exists an integer $\omega_{M} \geqslant 1$ such that, for all $u \in M$, the element $u^{\omega_{M}}$ is idempotent. Moreover, the element $u^{\omega_{M}}$ is the unique idempotent generated by $u$. Usually, the monoid $M$ is clear from the context and thus, we simply write $\omega$ instead of $\omega_{M}$. This leads to the following definition. An $\omega$-term over a finite alphabet $X$ is either a word in $X^{*}$, or of the form $t^{\omega}$ for some $\omega$-term $t$, or the concatenation $t_{1} t_{2}$ of two $\omega$-terms $t_{1}, t_{2}$. A homomorphism $\varphi: X^{*} \rightarrow M$ to a finite monoid $M$ uniquely extends to $\omega$-terms over $X$ by setting $\varphi\left(t^{\omega}\right)=\varphi(t)^{\omega_{M}}$. Let $u, v$ be two $\omega$-terms over $X$. A finite monoid $M$ satisfies the identity $u=v$ if $\varphi(u)=\varphi(v)$ for all homomorphisms $\varphi: X^{*} \rightarrow M$. The class of finite monoids satisfying the identity $u=v$ is denoted by $\llbracket u=v \rrbracket$. For all $\omega$-terms $u, v$, the class $\llbracket u=v \rrbracket$ forms a variety. We need the following three varieties in this paper:

$$
\begin{aligned}
\mathbf{R} & =\llbracket(x y)^{\omega} x=(x y)^{\omega} \rrbracket, \\
\mathbf{L} & =\llbracket x(z x)^{\omega}=(z x)^{\omega} \rrbracket, \\
\mathbf{W} & =\llbracket(x y)^{\omega} x(z x)^{\omega}=(x y)^{\omega}(z x)^{\omega} \rrbracket .
\end{aligned}
$$

A monoid is in $\mathbf{R}$ if and only if it is $\mathscr{R}$-trivial. Symmetrically, a monoid is in $\mathbf{L}$ if and only if it is $\mathscr{L}$-trivial. The aim of this paper is to give a new proof of Almeida and Azevedo's result $\mathbf{R} \vee \mathbf{L}=\mathbf{W}$. The inclusion $\mathbf{R} \vee \mathbf{L} \subseteq \mathbf{W}$ is trivial since $\mathbf{R} \cup \mathbf{L} \subseteq \mathbf{W}$ and $\mathbf{W}$ is a variety.

\section{Congruences}

In this section, we introduce the main combinatorial tool for our proof. It is a family of congruences $\equiv_{n}$ on $A^{*}$ for some finite alphabet $A$ such that $A^{*} / \equiv_{n} \in \mathbf{R} \vee \mathbf{L}$ for all integers $n \geqslant 0$, see Lemma 2 below. As a first step towards the definition of $\equiv_{n}$ we need to introduce an asymmetric, weaker congruence $\equiv_{n}^{\mathscr{R}}$. Let $u, v \in A^{*}$. We let $u \equiv_{0}^{\mathscr{R}} v$ if $\alpha(u)=\alpha(v)$. For $n \geqslant 0$, we let $u \equiv_{n+1}^{\mathscr{R}} v$ if the following conditions hold:

1. $\alpha(u)=\alpha(v)$,

2. for all factorizations $u=u_{1} a u_{2}$ and $v=v_{1} a v_{2}$ with $a \in A \backslash\left(\alpha\left(u_{1}\right) \cup \alpha\left(v_{1}\right)\right)$ we have $u_{1} \equiv_{n}^{\mathscr{R}} v_{1}$ and $u_{2} \equiv_{n}^{\mathscr{R}} v_{2}$, and 
3. for all factorizations $u=u_{1} a u_{2}$ and $v=v_{1} a v_{2}$ with $a \in A \backslash\left(\alpha\left(u_{2}\right) \cup \alpha\left(v_{2}\right)\right)$ we have $u_{1} \equiv_{n}^{\mathscr{R}} v_{1}$.

By a straightforward verification we see that $\equiv_{n}^{\mathscr{R}}$ is an equivalence relation. The factorization $u_{1} a u_{2}$ with $a \in A \backslash \alpha\left(u_{1}\right)$ is unique. Therefore, induction on $n$ shows that the index of $\equiv_{n}^{\mathscr{R}}$ is finite. If $u \equiv_{n+1}^{\mathscr{R}} v$, then $u \equiv_{n}^{\mathscr{R}} v$. Moreover, if $u \equiv_{n}^{\mathscr{R}} v$ and $a \in A$, then $a u \equiv_{n}^{\mathscr{R}} a v$ and $u a \equiv_{n}^{\mathscr{R}} v a$. Therefore, the relation $\equiv_{n}^{\mathscr{R}}$ is a finite index congruence on $A^{*}$.

Lemma 1 For every finite alphabet $A$ and every integer $n \geqslant 0$ we have $A^{*} / \equiv_{n}^{\mathscr{R}} \in \mathbf{R}$.

Proof: It suffices to show $(x y)^{n+1} x \equiv_{n}^{\mathscr{R}}(x y)^{n+1}$ for all words $x, y \in A^{*}$. We note that for $y=1$ this yields $x^{n+2} \equiv_{n}^{\mathscr{R}} x^{n+1}$. The proof is by induction on $n$. For $n=0$, the claim is true since $\alpha(x y x)=\alpha(x y)$. Let now $n>0$. As before, $\alpha\left((x y)^{n+1} x\right)=\alpha\left((x y)^{n+1}\right)$. Suppose $(x y)^{n+1} x=u_{1} a u_{2}$ and $(x y)^{n+1}=v_{1} a v_{2}$ for $a \in A \backslash\left(\alpha\left(u_{1}\right) \cup \alpha\left(v_{1}\right)\right)$. Then $u_{1}=v_{1}$ and both are proper prefixes of $x y$. Thus $u_{2}=p(x y)^{n} x$ and $v_{2}=p(x y)^{n}$ for some $p \in A^{*}$. By induction $(x y)^{n} x \equiv_{n-1}^{\mathscr{R}}(x y)^{n}$ and hence, $u_{2} \equiv_{n}^{\mathscr{R}} v_{2}$.

Suppose now $(x y)^{n+1} x=u_{1} a u_{2}$ and $(x y)^{n+1}=v_{1} a v_{2}$ for $a \in A \backslash\left(\alpha\left(u_{2}\right) \cup \alpha\left(v_{2}\right)\right)$. Then $a v_{2}$ is a suffix of $x y$ and $a u_{2}$ is a suffix of $y x$. We can therefore write $v_{1}=(x y)^{n} p^{\prime}$ for some prefix $p^{\prime}$ of $x y$. Similarly, $u_{1}=(x y)^{k} p$ for some $k \in\{n, n+1\}$ and some prefix $p$ of $x y$, i.e., we have $p q=x y$ for some $q \in A^{*}$. By induction, we have $(x y)^{n+1} \equiv_{n-1}^{\mathscr{R}}(x y)^{n}$ and thus $(x y)^{n+1} p \equiv_{n-1}^{\mathscr{R}}(x y)^{n} p$. We can therefore assume $k=n$. Without loss of generality, let $|p| \leqslant\left|p^{\prime}\right|$, i.e., $p^{\prime}=p s$ for some $s \in A^{*}$. It follows

$$
u_{1}=(p q)^{n} p \quad \text { and } \quad v_{1}=(p q)^{n} p s .
$$

Since $p^{\prime}=p s$ is a prefix of $x y=p q$, the word $s$ is a prefix of $q$. In particular, there exists $t \in A^{*}$ such that $q p=s t$. This yields

$$
u_{1}=p(s t)^{n} \quad \text { and } \quad v_{1}=p(s t)^{n} s .
$$

By induction, $(s t)^{n} \equiv_{n-1}^{\mathscr{R}}(s t)^{n} s$ and thus $u_{1} \equiv_{n-1}^{\mathscr{R}} v_{1}$. This shows $(x y)^{n+1} x \equiv_{n}^{\mathscr{R}}(x y)^{n+1}$ which concludes the proof.

There is a left-right symmetric congruence $\equiv_{n}^{\mathscr{L}}$ on $A^{*}$. It can be defined by setting $u \equiv_{n}^{\mathscr{L}} v$ if and only if $u^{\rho} \equiv{ }_{n}^{\mathscr{R}} v^{\rho}$. Here, $u^{\rho}=a_{n} \cdots a_{1}$ is the reversal of the word $u=a_{1} \cdots a_{n}$ with $a_{i} \in A$. It satisfies $A^{*} / \equiv_{n}^{\mathscr{L}} \in \mathbf{L}$ for every $n \geqslant 0$. We define $u \equiv_{n} v$ if and only if both $u \equiv_{n}^{\mathscr{R}} v$ and $u \equiv_{n}^{\mathscr{L}} v$. The following lemma puts together some properties of the finite index congruence $\equiv_{n}$.

Lemma 2 For every finite alphabet $A$ and every integer $n \geqslant 0$ the following properties hold:

1. $A^{*} / \equiv_{n} \in \mathbf{R} \vee \mathbf{L}$.

2. If $u_{1} a u_{2} \equiv_{n+1} v_{1} a v_{2}$ for $a \in A \backslash\left(\alpha\left(u_{1}\right) \cup \alpha\left(v_{1}\right)\right)$, then $u_{1} \equiv_{n}^{\mathscr{R}} v_{1}$ and $u_{2} \equiv_{n} v_{2}$.

3. If $u_{1} a u_{2} \equiv_{n+1} v_{1} a v_{2}$ for $a \in A \backslash\left(\alpha\left(u_{2}\right) \cup \alpha\left(v_{2}\right)\right)$, then $u_{1} \equiv_{n} v_{1}$ and $u_{2} \equiv_{n}^{\mathscr{L}} v_{2}$.

Proof: ' 11 ': We have $A^{*} / \equiv_{n} \in \mathbf{R} \vee \mathbf{L}$ since it is a submonoid of $\left(A^{*} / \bar{\equiv}_{n}^{\mathscr{R}}\right) \times\left(A^{*} / \equiv_{n}^{\mathscr{L}}\right)$, and $A^{*} / \equiv_{n}^{\mathscr{R}} \in \mathbf{R}$ and $A^{*} / \equiv_{n}^{\mathscr{L}} \in \mathbf{L}$ by Lemma 1 and its left-right dual. The properties ' 2 ' ' and ' 3 ' ' trivially follow from the definition of $\equiv_{n}$. 


\section{An Equation for the Join}

The goal of this section is to prove $\mathbf{W} \subseteq \mathbf{R} \vee \mathbf{L}$. By Lemma 2 it suffices to show that for every $A$-generated monoid $M \in \mathbf{W}$ there exists an integer $n \geqslant 0$ such that $M$ is a quotient of $A^{*} / \equiv_{n}$. The outline of the proof is as follows. First, in Lemma 3, we give a substitution rule valid in $\mathbf{W}$. Then, in Lemma 5, we show that $\equiv_{n}$-equivalence allows a factorization satisfying the premise for applying this substitution rule; this relies on a property of $\mathbf{W}$ shown in Lemma 4 . Finally, in Theorem 6 all the ingredients are put together.

Lemma 3 Let $M \in \mathbf{W}$ and let $u, v, x \in M$. If $u \mathscr{R} u x$ and $v \mathscr{L} x v$, then $u x v=u v$.

Proof: Since $u \mathscr{R} u x$ and $v \mathscr{L} x v$, there exist $y, z \in M$ with $u=u x y$ and $v=z x v$. In particular, we have $u=u(x y)^{\omega}$ and $v=(z x)^{\omega} v$. By $M \in \mathbf{W}$ we conclude $u x v=u(x y)^{\omega} x(z x)^{\omega} v=u(x y)^{\omega}(z x)^{\omega} v=u v$.

We will apply the previous lemma as follows. Let $M \in \mathbf{W}$ and $u, v, s, t \in M$ such that $u \mathscr{R} u s \mathscr{R} u t$ and $v \mathscr{L} s v \mathscr{L} t v$. Then $u s v=u t v$ since $u s v=u v$ and $u t v=u v$ by Lemma 3 The $\mathscr{R}$-equivalences and $\mathscr{L}$-equivalences for being able to apply this substitution rule are established in Lemma5 5 Before, we give a simple property of $\mathbf{W}$. It is the link between Green's relations and the congruence $\equiv_{n}$.

Lemma 4 Let $M \in \mathbf{W}$ and let $u, v, a \in M$. If $u \mathscr{R} v \mathscr{R} v a$, then $u \mathscr{R} u a$. If $u \mathscr{L} v \mathscr{L}$ av, then $u \mathscr{L}$ au.

Proof: Since $u \mathscr{R} v$ and $u \mathscr{R} v a$, there exist $x, y \in M$ with $v=u x$ and $u=v a y$. Now, $u=u x a y=$ $u(x a y)^{2 \omega+1}=u(x a y)^{\omega} x(a y x)^{\omega} a y=u(x a y)^{\omega}(a y x)^{\omega} a y=u(a y x)^{\omega} a y \in u a M$ where the fourth equality uses $M \in \mathbf{W}$. This shows $u M \subseteq u a M$ and thus $u \mathscr{R} u a$. The second implication is left-right symmetric.

The intuitive interpretation of the algebraic statement in Lemma4 4 is the following: For $M \in \mathbf{W}$ it only depends on the element $a$ and the $\mathscr{R}$-class of $u$ whether $u \mathscr{R} u a$ or not (but not on the element $u$ itself). The statement for $\mathscr{L}$-classes is analogous.

Lemma 5 Let $M \in \mathbf{W}$ and let $\varphi: A^{*} \rightarrow M$ be a homomorphism. If $u \equiv_{n} v$ for $n \geqslant 2|M|$, then there exist factorizations $u=a_{1} s_{1} \cdots a_{\ell-1} s_{\ell-1} a_{\ell}$ and $v=a_{1} t_{1} \cdots a_{\ell-1} t_{\ell-1} a_{\ell}$ with $a_{i} \in A$ and $s_{i}, t_{i} \in A^{*}$ and with $\ell \leqslant 2|M|$ such that for all $i \in\{1, \ldots, \ell-1\}$ we have:

$$
\begin{gathered}
\varphi\left(a_{1} s_{1} \cdots a_{i-1} s_{i-1} a_{i}\right) \mathscr{R} \varphi\left(a_{1} s_{1} \cdots a_{i} s_{i}\right) \mathscr{R} \varphi\left(a_{1} s_{1} \cdots a_{i-1} s_{i-1} a_{i} t_{i}\right), \\
\varphi\left(a_{i+1} t_{i+1} \cdots a_{\ell-1} t_{\ell-1} a_{\ell}\right) \mathscr{L} \varphi\left(t_{i} a_{i+1} \cdots t_{\ell-1} a_{\ell}\right) \mathscr{L} \varphi\left(s_{i} a_{i+1} t_{i+1} \cdots a_{\ell-1} t_{\ell-1} a_{\ell}\right) .
\end{gathered}
$$

Proof: To simplify notation, for some relation $\mathscr{G}$ on $M$ we write $u \mathscr{G} v$ for words $u, v \in A^{*}$ if $\varphi(u) \mathscr{G} \varphi(v)$. Consider the $\mathscr{R}$-factorization of $u$, i.e., let $u=b_{1} u_{1} \cdots b_{k} u_{k}$ with $b_{i} \in A$ such that

$$
\begin{array}{cl}
b_{1} u_{1} \cdots b_{i} \mathscr{R} b_{1} u_{1} \cdots b_{i} u_{i} & \text { for all } i \in\{1, \ldots, k\}, \\
b_{1} u_{1} \cdots b_{i} u_{i}>\mathscr{R} b_{1} u_{1} \cdots b_{i} u_{i} b_{i+1} & \text { for all } i \in\{1, \ldots, k-1\} .
\end{array}
$$

Similarly, let $v=v_{1} c_{1} \cdots v_{k^{\prime}} c_{k^{\prime}}$ be the $\mathscr{L}$-factorization of $v$, i.e., we have $c_{i} \in A$ and

$$
\begin{array}{cl}
c_{i} \cdots v_{k^{\prime}} c_{k^{\prime}} \mathscr{L} v_{i} c_{i} \cdots v_{k^{\prime}} c_{k^{\prime}} & \text { for all } i \in\left\{1, \ldots, k^{\prime}\right\}, \\
v_{i} c_{i} \cdots v_{k^{\prime}} c_{k^{\prime}}>\mathscr{L} c_{i-1} v_{i} c_{i} \cdots v_{k^{\prime}} c_{k^{\prime}} & \text { for all } i \in\left\{2, \ldots, k^{\prime}\right\} .
\end{array}
$$


We have $k, k^{\prime} \leqslant|M|$ because neither the number of $\mathscr{R}$-classes nor the number of $\mathscr{L}$-classes can exceed $|M|$. By Lemma 4, we have $b_{i} \notin \alpha\left(u_{i-1}\right)$ for all $i \in\{2, \ldots, k\}$ and $c_{i} \notin \alpha\left(v_{i+1}\right)$ for all $i \in\left\{1, \ldots, k^{\prime}-1\right\}$. We use these properties to convert the $\mathscr{R}$-factorization of $u$ to $v$ and to convert the $\mathscr{L}$-factorization of $v$ to $u$ : Let $v=b_{1} v_{1}^{\prime} \cdots b_{k} v_{k}^{\prime}$ such that $b_{i} \notin \alpha\left(v_{i-1}^{\prime}\right)$, and let $u=u_{1}^{\prime} c_{1} \cdots u_{k^{\prime}}^{\prime} c_{k^{\prime}}$ with $c_{i} \notin \alpha\left(u_{i+1}^{\prime}\right)$. These factorizations exist because $u \equiv_{n} v$; in particular, by Lemma 2 .

$$
\begin{aligned}
& u_{i} b_{i+1} u_{i+1} \cdots b_{k} u_{k} \equiv_{n-i} v_{i}^{\prime} b_{i+1} v_{i+1}^{\prime} \cdots b_{k} v_{k}^{\prime} \\
& v_{1} c_{1} \cdots v_{j-1} c_{j-1} v_{j} \equiv_{n-k^{\prime}-1+j} u_{1}^{\prime} c_{1} \cdots u_{j-1}^{\prime} c_{j-1} u_{j}^{\prime}
\end{aligned}
$$

for all $i \in\{1, \ldots k\}$ and $j \in\left\{1, \ldots, k^{\prime}\right\}$. Moreover, we see that $\alpha\left(u_{i}\right)=\alpha\left(v_{i}^{\prime}\right)$ and $\alpha\left(v_{j}\right)=\alpha\left(u_{j}^{\prime}\right)$.

We now show that the relative positions of the $b_{i}$ 's and $c_{j}$ 's in the above factorizations are the same in $u$ and $v$. Let $p$ be the position of $b_{i}$ in the $\mathscr{R}$-factorization of $u$ and let $q$ be the position of $c_{j}$ in the above factorization of $u$. Similarly, let $p^{\prime}$ be the position of $b_{i}$ in $v$ and let $q^{\prime}$ be the position of $c_{j}$ in $v$. First, suppose $p<q$. Let

$$
u=b_{1} u_{1} \cdots b_{i-1} u_{i-1} b_{i} u^{\prime} c_{j} u_{j+1}^{\prime} c_{j+1} \cdots u_{k^{\prime}}^{\prime} c_{k^{\prime}} .
$$

By an $i$-fold application of property '2]' in Lemma2 with $a \in\left\{b_{1}, \ldots, b_{i}\right\}$ (which is possible for $u$ ) we obtain $v=b_{1} v_{1}^{\prime} \cdots b_{i-1} v_{i-1}^{\prime} b_{i} z$ with $z \equiv_{n-i} u^{\prime} c_{j} u_{j+1}^{\prime} c_{j+1} \cdots u_{k^{\prime}}^{\prime} c_{k^{\prime}}$. By a $\left(k^{\prime}+1-j\right)$-fold application of property ' 3 ' in Lemma 2 with $a \in\left\{c_{k^{\prime}}, \ldots, c_{j}\right\}$ (which is possible for the word $u^{\prime} c_{j} u_{j+1}^{\prime} c_{j+1} \cdots u_{k^{\prime}}^{\prime} c_{k^{\prime}}$ ) we obtain $z=v^{\prime} c_{j} v_{j+1} c_{j+1} \cdots v_{k^{\prime}} c_{k^{\prime}}$. Thus

$$
v=b_{1} v_{1}^{\prime} \cdots b_{i-1} v_{i-1}^{\prime} b_{i} v^{\prime} c_{j} v_{j+1} c_{j+1} \cdots v_{k^{\prime}} c_{k^{\prime}}
$$

showing that $p^{\prime}<q^{\prime}$. Symmetrically, one shows that $p^{\prime}<q^{\prime}$ implies $p<q$. We conclude $p<q$ if and only if $p^{\prime}<q^{\prime}$. Similarly, we have $p=q$ if and only if $p^{\prime}=q^{\prime}$. It follows that the relative order of the $b_{i}$ 's and $c_{j}$ 's in $u$ and $v$ is the same. By factoring $u$ and $v$ at all $b_{i}$ 's and $c_{j}$ 's, we obtain $u=a_{1} s_{1} \cdots a_{\ell-1} s_{\ell-1} a_{\ell}$ and $v=a_{1} t_{1} \cdots a_{\ell-1} t_{\ell-1} a_{\ell}$ with $a_{i} \in A$ and $\ell \leqslant k+k^{\prime} \leqslant 2|M|$.

We have $a_{1} s_{1} \cdots a_{i-1} s_{i-1} a_{i} \mathscr{R} a_{1} s_{1} \cdots a_{i-1} s_{i-1} a_{i} s_{i}$ since the factorization $u=a_{1} s_{1} \cdots a_{\ell-1} s_{\ell-1} a_{\ell}$ is a refinement of the $\mathscr{R}$-factorization. Note that we cannot assume $\alpha\left(s_{i}\right)=\alpha\left(t_{i}\right)$. But each $t_{i}$ is a factor of some $v_{j}^{\prime}$, and at the same time $s_{i}$ is a factor of $u_{j}$. More precisely, there exists $m \leqslant i$ such that

$$
b_{1} v_{1}^{\prime} \cdots b_{j-1} v_{j-1}^{\prime} b_{j}=a_{1} t_{1} \cdots a_{m-1} t_{m-1} a_{m} \text { and } t_{m} a_{m+1} \cdots t_{i-1} a_{i} t_{i} \text { is a prefix of } v_{j}^{\prime} .
$$

Furthermore, $s_{m} a_{m+1} \cdots s_{i-1} a_{i} s_{i}$ is a prefix of $u_{j}$. Now, $\alpha\left(t_{i}\right) \subseteq \alpha\left(v_{j}^{\prime}\right)=\alpha\left(u_{j}\right)$ and, by Lemma 4 , for all words $z$ with $\alpha(z) \subseteq \alpha\left(u_{j}\right)$ we have $a_{1} s_{1} \cdots a_{i-1} s_{i-1} a_{i} \mathscr{R} a_{1} s_{1} \cdots a_{i-1} s_{i-1} a_{i} z$. Symmetrically we see $a_{i+1} t_{i+1} \cdots a_{\ell-1} t_{\ell-1} a_{\ell} \mathscr{L} t_{i} a_{i+1} \cdots t_{\ell-1} a_{\ell} \mathscr{L} s_{i} a_{i+1} t_{i+1} \cdots a_{\ell-1} t_{\ell-1} a_{\ell}$.

\section{Theorem 6 (Almeida/Azevedo, 1989 [2])}

$$
\mathbf{R} \vee \mathbf{L}=\llbracket(x y)^{\omega} x(z x)^{\omega}=(x y)^{\omega}(z x)^{\omega} \rrbracket
$$

Proof: The inclusion $\mathbf{R} \vee \mathbf{L} \subseteq \mathbf{W}$ is trivial since $\mathbf{R} \cup \mathbf{L} \subseteq \mathbf{W}$ and $\mathbf{W}$ is a variety of finite monoids. Let $M \in \mathbf{W}$ be generated by $A$, and let $\varphi: A^{*} \rightarrow M$ be the homomorphism induced by $A \subseteq M$. Let $n=2|M|$ and 
suppose $u \equiv_{n} v$. Let $u=a_{1} s_{1} \cdots a_{\ell-1} s_{\ell-1} a_{\ell}$ and $v=a_{1} t_{1} \cdots a_{\ell-1} t_{\ell-1} a_{\ell}$ be the factorizations from Lemma5. Applying Lemma 3 repeatedly, we get

$$
\begin{aligned}
\varphi(v) & =\varphi\left(a_{1} t_{1} a_{2} t_{2} \cdots a_{\ell-2} t_{\ell-2} a_{\ell-1} t_{\ell-1} a_{\ell}\right) \\
& =\varphi\left(a_{1} s_{1} a_{2} t_{2} \cdots a_{\ell-2} t_{\ell-2} a_{\ell-1} t_{\ell-1} a_{\ell}\right) \\
& =\varphi\left(a_{1} s_{1} a_{2} s_{2} \cdots a_{\ell-2} t_{\ell-2} a_{\ell-1} t_{\ell-1} a_{\ell}\right) \\
& \vdots \\
= & \varphi\left(a_{1} s_{1} a_{2} s_{2} \cdots a_{\ell-2} s_{\ell-2} a_{\ell-1} t_{\ell-1} a_{\ell}\right) \\
= & \varphi\left(a_{1} s_{1} a_{2} s_{2} \cdots a_{\ell-2} s_{\ell-2} a_{\ell-1} s_{\ell-1} a_{\ell}\right)=\varphi(u) .
\end{aligned}
$$

Note that the substitution rules $t_{i} \rightarrow s_{i}$ are $\varphi$-invariant only when applied from left to right. This shows that $M$ is a quotient of $A^{*} / \equiv_{n}$, and the latter is in $\mathbf{R} \vee \mathbf{L}$ by Lemma 2. Thus $M \in \mathbf{R} \vee \mathbf{L}$.

\section{Acknowledgements}

We thank the anonymous referees for several suggestions which helped to improve the presentation of the paper, and we gratefully acknowledge the support by the German Research Foundation (DFG) under grant DI $435 / 5-1$.

\section{References}

[1] J. Almeida. Finite Semigroups and Universal Algebra. World Scientific, 1994.

[2] J. Almeida and A. Azevedo. The join of the pseudovarieties of $\mathscr{R}$-trivial and $\mathscr{L}$-trivial monoids. J. Pure Appl. Algebra, 60:129-137, 1989.

[3] Th. Colcombet. Green's relations and their use in automata theory. In LATA 2011, volume 6638 of LNCS, pages 1-21. Springer, 2011.

[4] S. Eilenberg. Automata, Languages, and Machines, volume B. Academic Press, 1976.

[5] J. A. Green. On the structure of semigroups. Ann. Math. (2), 54:163-172, 1951.

[6] O. Klíma. Piecewise testable languages via combinatorics on words. Discrete Math., 311(20):2124-2127, 2011.

[7] R. König. Reduction algorithms for some classes of aperiodic monoids. RAIRO, Inf. Théor., 19(3):233-260, 1985.

[8] M. Kufleitner and A. Lauser. Languages of dot-depth one over infinite words. In LICS 2011, pages 23-32. IEEE Computer Society, 2011.

[9] J.-É. Pin. Varieties of Formal Languages. North Oxford Academic, 1986.

[10] J. Reiterman. The Birkhoff theorem for finite algebras. Algebra Univers., 14:1-10, 1982.

[11] M. P. Schützenberger. On finite monoids having only trivial subgroups. Inf. Control, 8:190-194, 1965.

[12] I. Simon. Piecewise testable events. In Autom. Theor. Form. Lang., 2nd GI Conf., volume 33 of LNCS, pages 214-222. Springer, 1975. 